\title{
MR Imaging of the Superior Cervical Ganglion and Inferior Ganglion of the Vagus Nerve: Structures That Can Mimic Pathologic Retropharyngeal Lymph Nodes
}

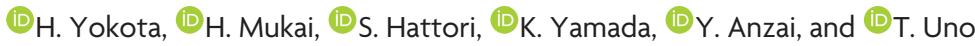

\begin{abstract}
BACKGROUND AND PURPOSE: The superior cervical ganglion and inferior ganglion of the vagus nerve can mimic pathologic retropharyngeal lymph nodes. We studied the cross-sectional anatomy of the superior cervical ganglion and inferior ganglion of the vagus nerve to evaluate how they can be differentiated from the retropharyngeal lymph nodes.
\end{abstract}

MATERIALS AND METHODS: This retrospective study consists of 2 parts. Cohort 1 concerned the signal intensity of routine neck MR imaging with 2D sequences, apparent diffusion coefficient, and contrast enhancement of the superior cervical ganglion compared with lymph nodes with or without metastasis in 30 patients. Cohort 2 used 3D neurography to assess the morphology and spatial relationships of the superior cervical ganglion, inferior ganglion of the vagus nerve, and the retropharyngeal lymph nodes in 50 other patients.

RESULTS: All superior cervical ganglions had homogeneously greater enhancement and lower signal on diffusion-weighted imaging than lymph nodes. Apparent diffusion coefficient values of the superior cervical ganglion $\left(1.80 \pm 0.28 \times 10^{-3} \mathrm{~mm}^{2} / \mathrm{s}\right)$ were significantly higher than normal and metastatic lymph nodes $\left(0.86 \pm 0.10 \times 10^{-3} \mathrm{~mm}^{2} / \mathrm{s}, P<.001\right.$, and $\left.0.73 \pm 0.10 \times 10^{-3} \mathrm{~mm}^{2} / \mathrm{s}, P<.001\right)$. Ten and 13 of 60 superior cervical ganglions were hypointense on T2-weighted images and had hyperintense spots on both T1- and T2-weighted images, respectively. The latter was considered fat tissue. The largest was the superior cervical ganglion, followed in order by the retropharyngeal lymph node and the inferior ganglion of the vagus nerve $(P<.001$ to $P=.004)$. The highest at vertebral level was the retropharyngeal lymph nodes, followed, in order, by the inferior ganglion of the vagus nerve and the superior cervical ganglion $(P<.001$ to $P=.001)$. The retropharyngeal lymph node, superior cervical ganglion, and inferior ganglion of the vagus nerve formed a line from anteromedial to posterolateral.

CONCLUSIONS: The superior cervical ganglion and the inferior ganglion of the vagus nerve can be almost always differentiated from retropharyngeal lymph nodes on MR imaging by evaluating the signal, size, and position.

ABBREVIATIONS: CCAB = common carotid artery bifurcation; FS = fat-saturated; IGVN = inferior ganglion of the vagus nerve; RPLN = retropharyngeal lymph node; $S C G$ = superior cervical ganglion

$\mathrm{T}$ he autonomic nervous system is composed of 2 antagonistic sets of nerves: the sympathetic and parasympathetic nervous systems. The sympathetic efferent pathway starts at the posterolateral hypothalamic region and descends along the encephalic trunk to the intermediolateral horn cells of the thoracic spinal cord. The sympathetic nerves then form chain structures lying

Received April 27, 2017; accepted after revision August 28.

From Diagnostic Radiology and Radiation Oncology (H.Y., H.M., S.H., T.U.), Graduate School of Medicine, Chiba University, Chiba, Japan; Department of Radiology (K.Y.), Graduate School of Medical Science, Kyoto Prefectural University of Medicine, Kyoto, Japan; and Department of Radiology (Y.A.), University of Utah School of Medicine Health Sciences, Salt Lake City, Utah.

Please address correspondence to Hajime Yokota, MD, PhD, Diagnostic Radiology and Radiation Oncology, Graduate School of Medicine, Chiba University, 1-8-1, Inohana, Chuo-ku, Chiba City, Chiba, Japan, 260-8670; e-mail: yokotaha@gmail.com 三 Indicates article with supplemental on-line tables.

http://dx.doi.org/10.3174/ajnr.A5434 just lateral to the vertebral bodies. The structures of the sympathetic nervous system to date have not been characterized in detail with conventional neck imaging, to our knowledge.

The cervical sympathetic trunk lies on the prevertebral fascia medial to the carotid sheath and contains 3 interconnected ganglia: the stellate, middle cervical, and superior cervical ganglia. ${ }^{1-7}$ The stellate ganglion is formed by the lower 2 cervical and first thoracic segmental ganglia. The middle cervical ganglion is the smallest one of the 3 ganglia. The superior cervical ganglia are elongated and cylindric and are the largest of the 3 ganglia (Fig 1). The size and location of the sympathetic ganglia have many variations. Although the stellate and middle cervical ganglia are occasionally divided into several parts, ${ }^{1,3,4-6}$ the superior cervical ganglia are invariably detected bilaterally. ${ }^{4-6}$ Imaging anatomy of the superior and stellate ganglia have only been reported in a few articles, ${ }^{8-11}$ whereas the middle cervical ganglion has not been described. 

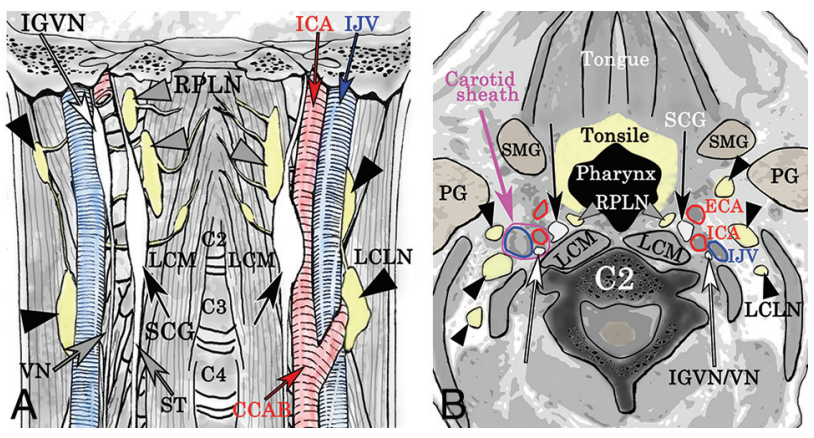

FIG 1. The coronal $(A)$ and axial $(B)$ views around the superior cervical ganglion according to anatomic reports show the superior cervical ganglion (black arrow), the inferior ganglion of the vagus nerve (white arrow), and the retropharyngeal lymph node (gray arrowheads). IJV indicates inferior jugular vein; ECA, external carotid artery; LCLN, lateral cervical lymph node (black arrowheads); LCM, longus capitis muscle; PG, parotid gland; SMG, submandibular gland; ST, sympathetic trunk; VN, vagus nerve. The magenta circle indicates the right carotid sheath.

In a clinical setting such as cancer staging of the head and neck, it is problematic that the superior cervical ganglia (SCGs) can mimic retropharyngeal lymph nodes (RPLNs). In anatomic reports, ${ }^{1-7}$ the SCGs are located at the side of the longus capitis or longus colli muscle around the C2 level. The lateral retropharyngeal lymph nodes are also distributed in the same region, extending from the level of the nasopharynx to the hyoid bone (the skull base to C3). The inferior ganglion of the vagus nerve (IGVN) is also along the internal carotid artery at approximately the same region, though there has been no imaging report of the IGVN, to our knowledge.

It is critical to focus on the SCG when performing interventional procedures of the neck, including open surgery and percutaneous local opioid anesthesia. Damage to this system can cause Horner syndrome. There is, however, no clear-cut method to differentiate SCGs from RPLNs and IGVNs during routine clinical imaging studies of the neck.

The purposes of this study were to describe the imaging anatomy of the SCG and IGVN and to propose methods to differentiate them from the RPLN. To accomplish this task, we performed studies on 2 separate cohorts. First, we investigated the signal intensity of these structures on routine $2 \mathrm{D}$ neck MR imaging. The second part of the study was conducted with MR neurography ${ }^{12-15}$ to assess the morphology and spatial relationships of the SCG, IGVN, and RPLN.

\section{MATERIALS AND METHODS}

This retrospective study was approved by the institutional review board of Chiba University Hospital without additional informed consent. We searched cases from January 2016, retrospectively, according to the inclusion criteria listed below until reaching the required number of cases.

\section{Power Analysis}

Power analysis for paired and unpaired $t$ tests among the SCG, IGVN, and RPLN indicated that 44 and 86 samples would be needed to detect type I error rates $(\alpha)$ of .05 to achieve a power of $90 \%$, considering effect size as a medium effect of .5. A sample size
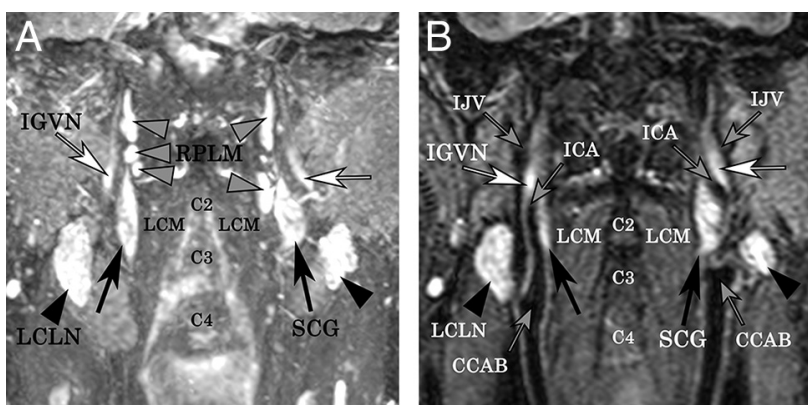

FIG 2. We assessed bilateral superior cervical ganglia (black arrows), the inferior ganglion of the vagus nerve (white arrows), and the retropharyngeal lymph nodes (gray arrowheads) using 3D-STIR (A, maximum-intensity-projection image; $B$, a section of original coronal images). The artery and vein clearly demonstrate flow voids. IJV indicates inferior jugular vein; LCLN, lateral cervical lymph node (black arrowhead); LCM, longus capitis muscle.

of 60 and 100 SCGs and IGVNs of 30 and 50 cases were chosen for cohorts 1 and 2, respectively.

\section{Structure Identification}

Two board-certified neuroradiologists (with 12 and 10 years' experience) independently assessed the SCGs, IGVNs, and RPLNs and later evaluated them with consensus when necessary.

On the basis of previous anatomic reports (Fig 1), ${ }^{1-7}$ we defined the SCGs as structures located symmetrically along the lateral edges of the longus capitis or longus colli muscle (Fig 2). An elongated cylindric or fusiform structure was searched cranially from the level of the common carotid artery bifurcation (CCAB).${ }^{16}$ A tapering shape of the superior and/or inferior poles, which presumably reflected the transitional zone between SCGs and the sympathetic trunk, was also used as a reference.

During the processes of identifying the SCG, there were objects with an MR imaging appearance like that of the SCG along the jugular vein, and we hypothesized that they represent the inferior ganglion of the vagus nerve. They had roughly the same size as the SCG as reported in previous anatomic studies (Fig 1). ${ }^{1-7}$

Nodular structures located from the suprahyoid retropharyngeal space to the skull base were identified to detect RPLNs. Lateral cervical lymph nodes were also detected.

\section{Cohort 1: Signal Intensities of the SCG and Lymph Nodes Using Routine Neck MR Imaging}

Subjects 1. From February 2015 to January 2016, eighty-four patients underwent neck MR imaging for the evaluation of both benign and malignant neoplasms of the head and neck before treatment. Fifty-four of 84 patients were excluded from this cohort because of an insufficient FOV $(n=34)$, inappropriate imaging protocol $(n=9)$, remarkable artifacts $(n=6)$, or tumor invasion or cervical vertebral body metastases with invasion in the retropharyngeal space $(n=5)$. Finally, this cohort included 30 patients (mean age, $63.0 \pm 11.5$ years; 9 women, 21 men). Twenty of 30 MRIs were performed for oral or pharyngeal cancers. Eleven of the 20 patients had unilateral lymph node metastasis from oral cancers or pharyngeal cancers on the internal jugular chain. Lymph node metastasis was determined by either pathologic examinations or interval changes after treatment.

AJNR Am J Neuroradiol 39:170-76 Jan 2018 www.ajnr.org 
Imaging Protocol 1. Neck MRIs were performed with a 1.5T scanner (Signa HDxt; GE Healthcare, Milwaukee, Wisconsin) using the following parameters: axial T1WI (TR/TE $=500 / 8.2 \mathrm{~ms})$, axial and coronal T2WI (TR/TE $=3850-4075 / 85-88 \mathrm{~ms})$, axial fat-saturated (FS)-T2WI (TR/TE $=3850 / 85 \mathrm{~ms}$, squares estimation), axial and coronal gadolinium-enhanced FS-T1WI (TR/ $\mathrm{TE}=500-510 / 8.2-9.2 \mathrm{~ms}$, chemical shift selective suppression, FOV $=220 \times 220 \mathrm{~mm}$, resolution $=0.430 \times 0.430 \times 5.000 \mathrm{~mm}$, gap $=1.000 \mathrm{~mm})$, sagittal $3 \mathrm{D}$ gadolinium-enhanced FS-T1WI (TR/TE $=8.1 / 3.6 \mathrm{~ms}$, echo-spoiled gradient echo, squares estimation, $\mathrm{FOV}=240 \times 240 \mathrm{~mm}$, resolution $=0.938 \times 0.938 \times$ $0.700 \mathrm{~mm})$, and axial DWI (TR/TE $=7000 / 80.2 \mathrm{~ms}$, b-value $=0$ and $1000 \mathrm{~s} / \mathrm{cm}^{2}$, FOV $=280 \times 280 \mathrm{~mm}$, resolution $=1.094 \times$ $1.094 \times 5.000 \mathrm{~mm}$, gap $=1.000 \mathrm{~mm})$. All sequences used in-plane parallel imaging with an array spatial sensitivity encoding technique factor of 2 .

Evaluation 1. Signal intensities of SCGs were evaluated and compared with those of lymph nodes with consensus. Each SCG was compared with an ipsilateral lymph node that was $\geq 5 \mathrm{~mm}$ and the largest in the scanned area. Apparent diffusion coefficient values of these structures were also measured. Metastatic and normal lymph nodes were evaluated separately.

\section{Cohort 2: Morphology and Spatial Relationships of the SCG, IGVN, and RPLN on Neurography}

Subjects 2. During the study period from September 2011 to January 2016, three hundred nineteen patients underwent MR neurography using 3D-STIR imaging to examine neuropathy or trauma of the brachial plexus before treatment. Two hundred sixty-nine of 319 cases were excluded from this cohort because of clinical symptoms related to the sympathetic and parasympathetic nervous systems $(n=10)$ or pathology that may lead to peripheral nerve hypertrophy or atrophy, such as chronic inflammatory demyelinating polyneuropathy and amyotrophic lateral sclerosis $(n=259)$. Finally, this cohort included 50 of 319 (mean age, $46.8 \pm 19.9$ years; 23 women; 27 men). These 50 patients had no disease that might account for abnormal retropharyngeal lymph nodes.

Imaging Protocol 2. 3D-STIR was acquired with a 1.5T scanner (Achieva; Philips Healthcare, Best, the Netherlands). The parameters of the 3D-STIR were the following: TR/TE/TI $=1600 / 200 /$ $180 \mathrm{~ms}$, with coronal acquisitions along the cervical spine (FOV $=$ $380 \times 380 \mathrm{~mm}$, resolution $=0.742 \times 0.742 \times 1.200 \mathrm{~mm})$.

Evaluation 2. Two neuroradiologists tried detecting the SCG, IGVN, and RPLN independently. The length and width of each SCG, IGVN, and RPLN were measured by a single neuroradiologist. The ROI was drawn, and the volume of these structures was computed using Analysis of Functional Neuro Images (AFNI; http://afni.nimh.nih.gov/afni). The distance between the CCAB and the inferior pole of the SCG was also measured. The CCAB could be detected because arteries showed flow voids on 3D-STIR. The levels of the superior and posterior poles of the SCG, RPLN, and $\mathrm{CCAB}$ were defined by reference to the spinal column.

A probability map was created to reveal variations of SCGs. The location of SCGs was accessed relative to the location of 2 anatomic landmarks: the $\mathrm{C} 2$ transverse process and the CCAB.

\section{Statistical Analysis}

Power analysis was performed with $\mathrm{G}^{\star}$ Power 3.1.9.2 software (http:// www.softpedia.com/get/Science-CAD/G-Power.shtml). ${ }^{17}$ The other statistical analyses were performed with the SPSS 22.0 software package (IBM, Armonk New York). ADC values of the SCGs and lymph nodes were compared using a 2-tailed unpaired $t$ test. Regarding the morphology and location, comparisons between the right and left and among SCGs, RPLNs, and IGVNs were also performed with paired and unpaired $t$ tests for continuous variables and the Wilcoxon signed rank and MannWhitney $U$ tests for categoric data.

\section{RESULTS}

\section{Detectability and Interreader Agreement}

In cohort 1 , two radiologists identified bilateral SCGs and lymph nodes in all 30 cases. The lateral cervical lymph node was chosen for evaluation when RPLNs of $>5 \mathrm{~mm}$ were not identified. Interobserver agreement was achieved in 57 of 60 SCGs (95.0\%). Sixty bilateral SCGs were detected in all 30 cases with consensus. Fortynine normal and 11 metastatic lymph nodes were also identified.

In cohort 2, two radiologists identified 100 bilateral SCGs and 100 IGVNs in all 50 cases and 104 RPLNs in 40 of 50 cases $(0-5$ RPLNs were detected in each case). At the independent reading, both readers concurred in 99 of 100 SCGs (99.0\%), 96 of 100 IGVNs (96.0\%), and 100 of 104 RPLNs (96.2\%).

\section{Signal Intensity and Contrast Enhancement}

All SCGs had homogeneous, avid enhancement compared with normal and metastatic lymph nodes (Fig $3 A$ ). In contrast, it was difficult to differentiate SCGs from both normal and metastatic lymph nodes on the basis of the signal intensity of T1WI, T2WI, and FS-T2WI (Fig 3B, -C).

Some of the SCGs had 2 different types of spot or slit-shaped areas within them, which were not obvious in any of the lymph nodes. One was a hypointense area on T2WI and FS-T2WI and less evident on T1WI and gadolinium-enhanced FS-T1WI (Fig $4 A,-B)$. The other was a hyperintense area on T1WI and T2WI that disappeared on FS-T2WI and gadolinium-enhanced FST1WI, indicative of fat tissue (Fig 4C, -D). The hypointense and hyperintense areas were seen in $10(16.7 \%)$ and $13(21.7 \%)$ of 60 SCGs, respectively. In 1 SCG, both of these structures were found. The IGVNs could not be detected on routine neck MR imaging with confidence.

\section{ADC Values}

The signal intensities of all SCGs on DWI were lower than those of both normal and metastatic lymph nodes (less restricted diffusion, Fig $3 D$ ). ADC values of the SCGs and normal and metastatic lymph nodes were $1.80 \pm 0.28,0.86 \pm 0.10$, and $0.73 \pm 0.10 \times$ $10^{-3} \mathrm{~mm}^{2} / \mathrm{s}$, respectively. ADC values of each SCG were significantly higher than observed in normal and metastatic lymph nodes $(P<.001$ and $<.001)$. Metastatic lymph nodes showed higher signal intensity on DWI and lower ADC values $(P=.001$, more restricted) than those of normal lymph nodes. 

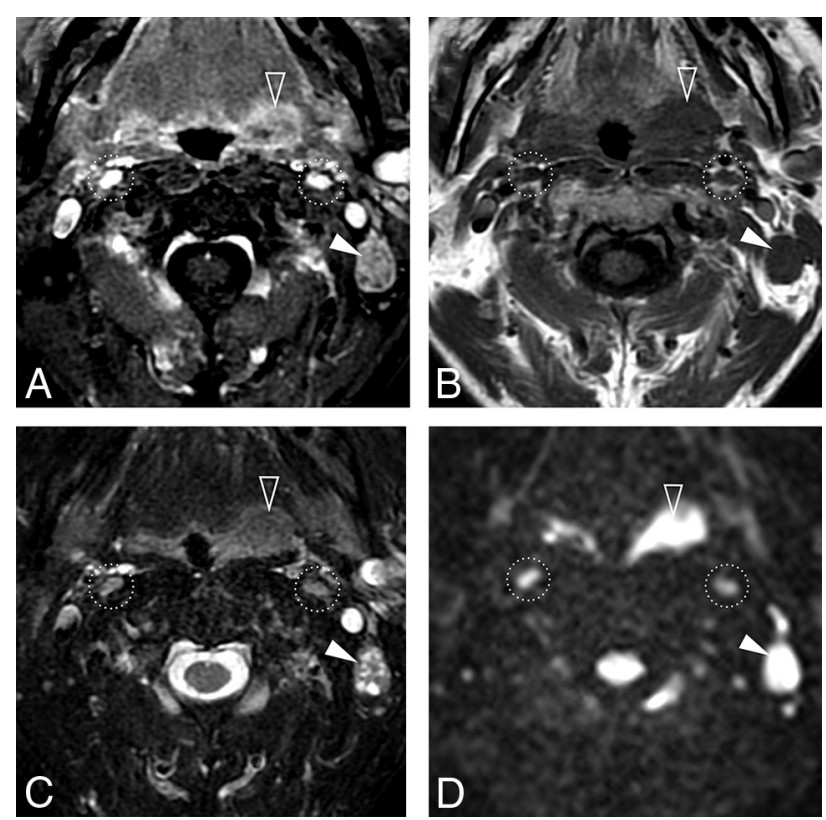

FIG 3. A 62-year-old man with oropharyngeal cancer with left lymph node metastasis. Bilateral superior cervical ganglia (circles), metastatic lymph node (arrowhead), and cancer (open arrowhead) are noted. Contrast-enhanced fat-saturated T1-wighted image $(A)$ shows stronger enhancement of the SCG than the metastatic lymph node. The T1-weighted image $(B)$ shows almost the same signals. Fat-saturated T2-weighted image (C) shows heterogeneous signal of the metastatic lymph node, whereas the SCG is homogeneous. Diffusion-weighted image shows that the signals of the SCGs are lower than those of the metastatic lymph node. ADC values of the right SCG, left SCG, and metastatic lymph nodes were $1.32,1.54$, and $0.90 \times 10^{-3} \mathrm{~mm}^{2} / \mathrm{s}$, respectively.
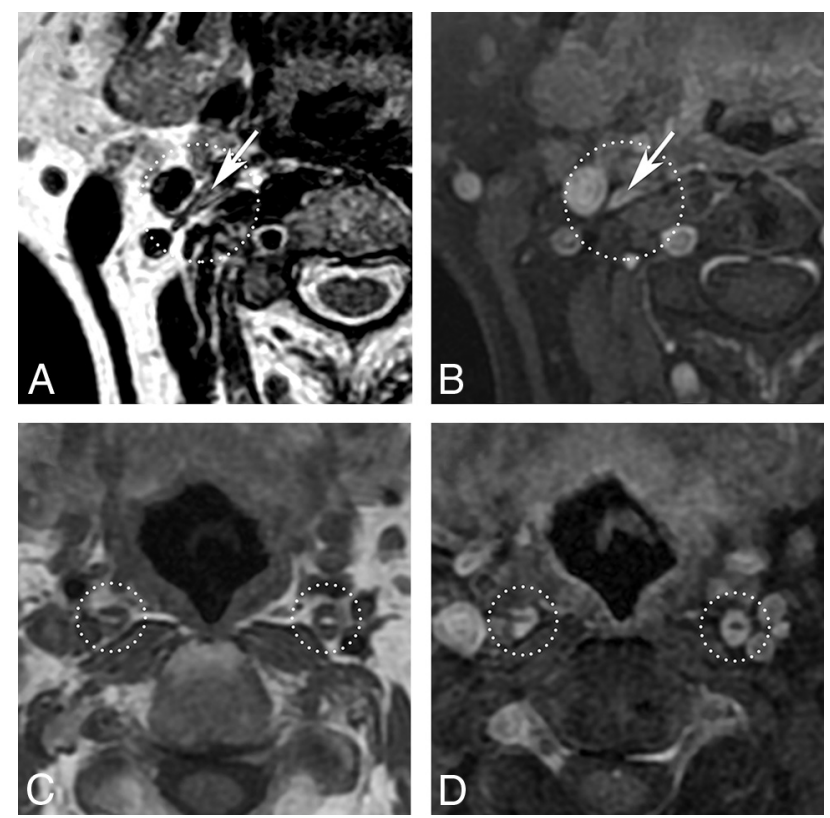

FIG 4. A 58-year-old woman with an intraganglionic hypointense spot (arrow) on the right on T2-weighted image ( $A)$. This spot is less evident (arrow) on the gadolinium-enhanced fat-saturated T7weighted image (B). T1-weighted images in a 65-year-old man show slit-shaped hyperintense areas in the bilateral superior cervical ganglia (C, circles). Signals of these regions are suppressed on the gadoliniumenhanced fat-saturated T7-weighted image (D).
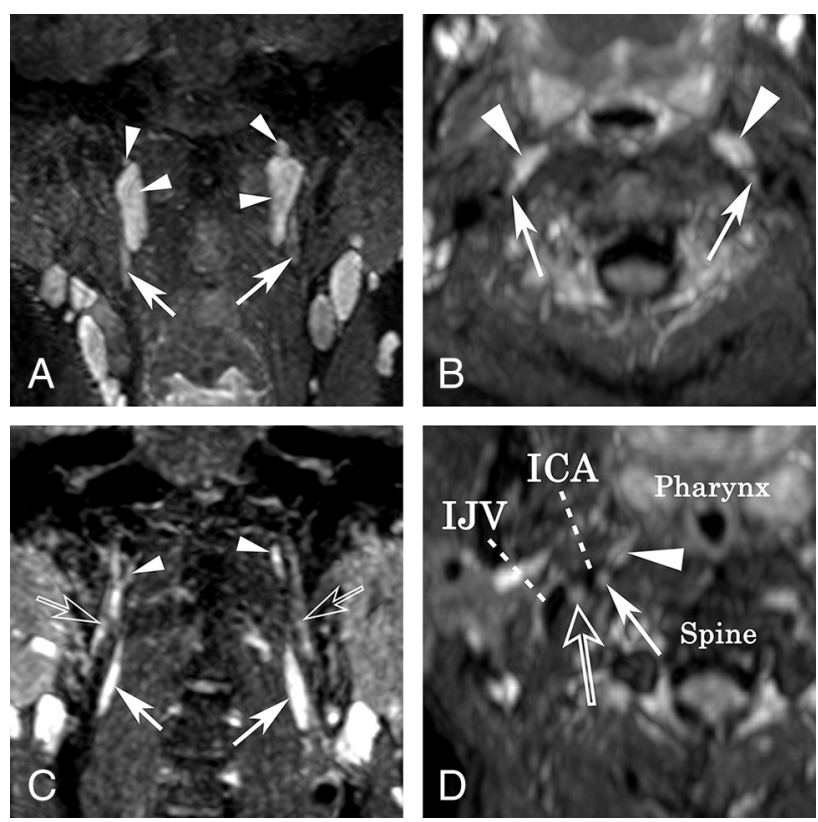

FIG 5. A, A 14-year-old adolescent with a prominent but normal RPLN. Coronal maximum-intensity-projection image shows the superior cervical ganglia (arrows) and the retropharyngeal lymph nodes (arrowheads). Each SCG was inferior to the RPLN. B, Reconstructed axial MIP image of 15-mm thickness shows that each SCG (arrows) is posterolateral to the RPLN (arrowheads). C, Coronal MIP image shows the SCG (arrows), inferior ganglia of vagus nerve (open arrows), and RPLN (arrowheads) in a 38-year-old man. The SCG is inferior to the IGVN. D, Reconstructed axial MIP image shows that the RPLN (arrowhead), SCG (arrow), internal carotid artery, IGVN (open arrow), and the internal jugular vein (IJV) form a line from anteromedial to posterolateral. $B$ and $D$, Blurring on the images is because the MIP was used to superimpose the SCG, IGVN, and RPLN. IJV indicates inferior jugular vein.

\section{Morphology}

There were no significant differences between the right and left sides or of all morphologic parameters and vertebral levels of SCGs and RPLNs (On-line Tables 1 and 2). Most SCGs were significantly larger than RPLNs $(P<.001 / P<.001$ on the right and left), though 26 RPLNs (25\%) had higher volume than SCGs (Fig $5 A$ ). IGVNs also showed symmetric morphology between the right and left sides (On-line Table 3). The volume of the IGVNs was smaller than that of the SCGs and RPLNs $(P<.001 / P<.001$, and $P=.004 / P=.018$, respectively).

\section{Spatial Relationships}

Ninety-three of 100 SCGs were located at the anteromedial or medial side of the internal carotid artery. Only 6 of 100 SCGs were located at the posterolateral side of the ICA, in which there was tortuosity of the vessels toward the medial side. The SCGs tended to be located caudal to the RPLNs $(P<.001 / P<.001)$, though 31 RPLNs (29.8\%) partially overlapped the SCGs on the anteroposterior projection. All RPLNs were located anteromedial to the SCGs (Fig 5B).

The IGVNs tended to be located cranial to the SCGs $(P<$ $.001)$. The center of the IGVNs tended to be located caudal to the RPLNs $(P=.001 / P=0.046)$, though the superior pole of the IGVNs had no difference of vertebral level $(P=.062 / P=.986)$. Compared with the SCG, the IGVNs were located cranial to the 

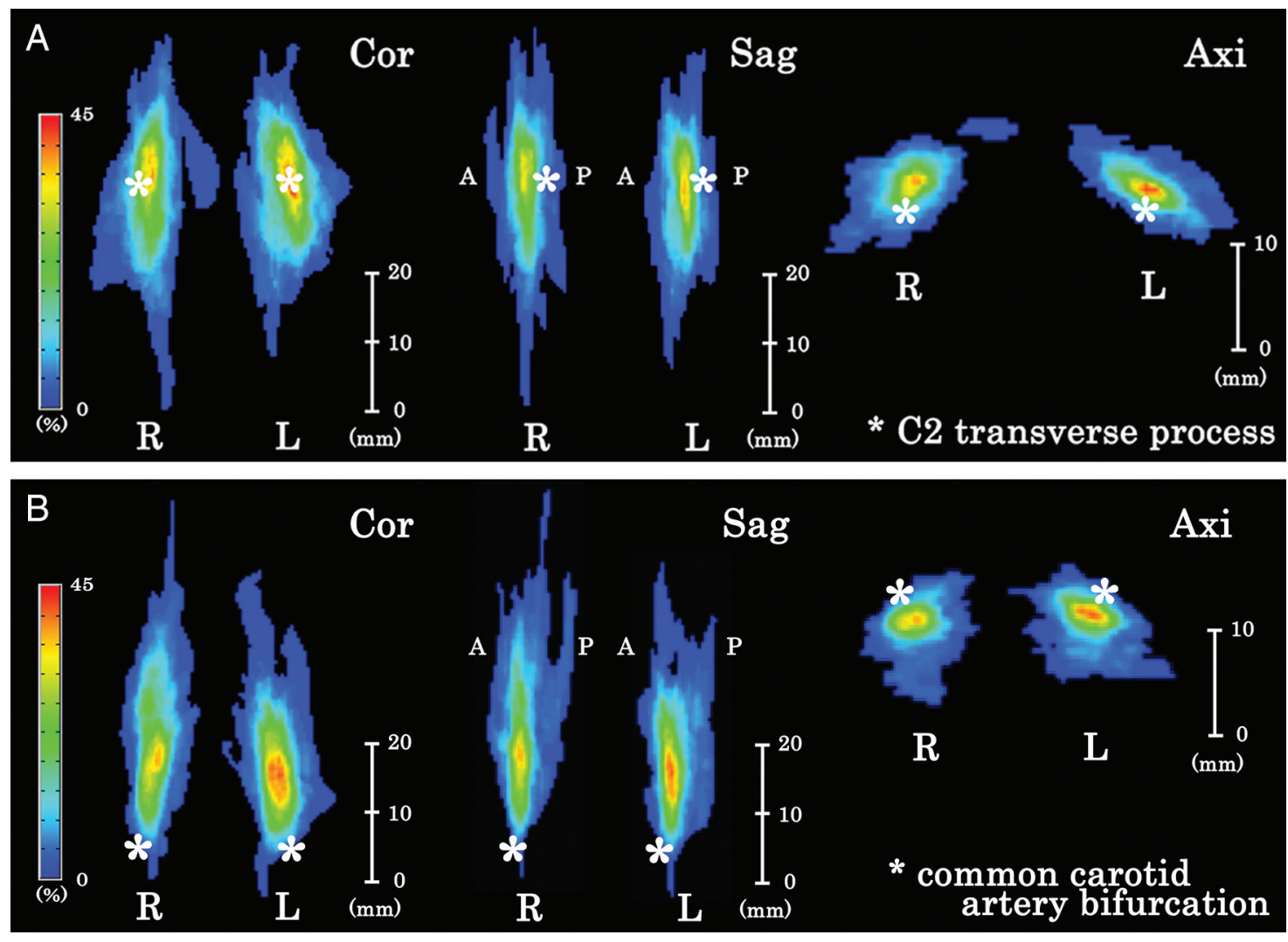

FIG 6. Probability maps of the superior cervical ganglion against the $\mathrm{C} 2$ transverse process $(A$, asterisk) and common carotid artery bifurcation ( $B$, asterisk). The SCG was located at the anterior side against the $C 2$ transverse process and superior and posterolateral to the CCAB. The location of bilateral SCGs is similar. R indicates right; L, left; Cor, coronal; Sag, sagittal; Axi, axial.

RPLNs $(P<.001 / P<.001)$. All IGVNs were located lateral or posterolateral to the SCGs and RPLNs.

\section{Probability Map of SCGs}

The probability map of SCGs showed them to be most commonly located in front of the $\mathrm{C} 2$ transverse process (Fig 6A) and superior to and posterolateral to the CCABs (Fig 6B). The maximum existence probabilities were $42 \%$ of the map (On-line Table 4 ). For example, $10 \%$ existence probability in this figure indicated that 10 of 100 SCGs existed in the area.

\section{DISCUSSION}

This is the first MR imaging analysis focused on identifying SCGs and IGVNs, to our knowledge. The SCG drew little attention in the past, and several previous reports mistakenly described it as retropharyngeal lymph node metastasis or tumor. ${ }^{18,19}$ The results of the current study indicate that MR imaging can be useful in identifying these structures and would potentially facilitate safer and more accurate planning of surgical and interventional procedures such as SCG blocks for facial pain.

RPLNs are usually less evident in elderly populations than in children. ${ }^{20}$ Because we assessed the MR imaging of elderly patients in cohort 1 , most of whom presented with oral or pharyngeal cancers, the detection of SCGs was comparatively straightforward because of less evident RPLNs. The enhancement and DWI characteristics were most useful for differentiation in the analysis. The SCG has a large number of capillary vessels around the ganglion cells ${ }^{8,21}$ and lacks a blood-nerve barrier. ${ }^{22}$ These features might cause avid enhancement of the SCGs. Although metastatic lymph nodes can be strongly enhanced due to angiogenesis by cancer cells, ${ }^{23}$ signals were often heterogeneous. Homogeneous enhancement was considered one of the characteristics of SCGs. The signal difference on DWI was likely due to differences in the ADC values because the signal intensity was visually about the same with conventional T2WI. ADC values can reflect histologic characteristics such as cell density. The SCG is constructed of ganglion cells, nerve fibers, vascular structures, and collagen fibers. ${ }^{8,21}$ By contrast, lymph nodes show low ADC values due to lymphocyte accumulation.

Intraganglionic hypointense spots on T2WI might be consistent with the 2 previous reports that concluded that they represent venules. ${ }^{8,9}$ Although this finding can be a characteristic feature of SCGs, metastatic lymph nodes were often heterogeneous in signal and might mimic this finding. In contrast, slits or spot-shaped fat tissue had not been previously described and were thought to be much more specific. The figure of T2WI presented by Loke et al ${ }^{8}$ appeared to have FS-T2WI, presumably due to signal intensity changes caused by the low body temperature of the corpus. Our assumption is that the fat tissue in the SCGs may represent spaces among the neural branches. SCGs have many branches: superior, lateral, medial, and anterior. The superior branches enter the cranial cavities along with the ICA. The gray rami of the lateral branches communicate with the upper 4 cervical spinal nerves, as well as with some of the cranial nerves such as the vagus and hypoglossal nerves. The medial branches are laryngopharyngeal 
and cardiac. The anterior branches are rami and those on the common and external carotid arteries. These branches may entrap fat tissue near the SCGs and mimic intraganglionic fat. Caution should be taken not to mistake fat tissue for lymph node hilum.

Cadaver studies have reported that the length and width of the SCGs are about $10-30$ and $5-8 \mathrm{~mm}$ and are located posterior to the ICA. ${ }^{1-7}$ The height of the SCGs varies in these reports from the level of C2-C3, just C2, just C4, and so forth. ${ }^{1-7}$ Although our results were mostly consistent with these cadaveric studies, there were some discrepancies. Our results showed that SCGs were located not posterior to but anteromedial or medial to the ICA in almost all cases. In addition, SCGs were widely distributed from $\mathrm{C} 1$ to $\mathrm{C} 5$ in our study. Cadaveric studies potentially present a risk of artifactual changes in location due to postmortem procedures and postmortem changes. Only MR imaging can reveal precise shapes and locations of the SCGs in vivo.

MR imaging allowed detection of bilateral SCGs in all cases. SCGs have elongated, cylindric, and fusiform shapes, and the longus capitis muscle and ICA were landmarks to detect them. The RPLNs were also located along the longus capitis muscle and had an elongated appearance along the body axis; therefore, the size and positional relationships were the most important factors in assessing them correctly. While many SCGs were, in general, longer than the RPLNs in the craniocaudal direction, some of the RPLNs were larger than the SCGs. In such cases, focusing on the relative positions will be of pivotal importance. SCGs are usually located caudal to the RPLNs. IGVNs also have a fusiform shape; however, once again, the location was the most important key to differentiating IGVNs and SCGs. The RPLNs were located at the anteromedial side of the SCGs, and IGVNs were located lateral or posterolateral to the SCGs. As a result, the RPLN, SCG, the ICA, and IGVN formed a line on an axial plane from anteromedial to posterolateral (Fig 5D). In addition, IGVNs might not cause confusion using routine neck MR imaging. Although we attempted to detect IGVNs, retrospectively, in cases of routine neck MR imaging, IGVNs were difficult to detect with confidence, presumably because the volume of the IGVNs was small and the signals due to vascular structures compromised their detection.

Probability mapping showed that the positional relationship had a large degree of variation. If the sites with maximum probability were visually checked, the SCGs could not be detected in more than half of the cases. The SCGs are one of the targets for ganglionic local opioid injection for migraine, trigeminal neuralgia, postherpetic neuralgia, facial pain, complex regional pain syndrome, pain-associated depression, and vasospasms following subarachnoid hemorrhage. ${ }^{24-26}$ Blinded or fluoroscopically guided approaches have been described. ${ }^{24,27,28}$ These blinded techniques were expected to have a high risk of inaccurate localization of the targets. A potential risk of vertebral artery puncture and epi-/subdural injection has also been demonstrated. ${ }^{26}$ Sonography-guided identification of the SCGs could be a potentially reliable technique, though this has only been validated in cadavers. ${ }^{25}$ For the sonography study, the CCAB and transverse processes of the spinal bones were used as landmarks. The probability maps created do not favor one landmark over the other because the probability densities of SCGs showed no evident difference between the 2 maps. We believe that MR imaging-guided in- tervention might be one of the options for failed pain control under a blinded injection. Although an SCG block under MR imaging still requires controlled studies with blinded injection, ${ }^{26,29} \mathrm{MR}$ imaging may have a potential role in identifying the precise location of SCGs.

Limitations of this study include a lack of direct clinicopathologic correlation of the identified ganglia by either an operation or postmortem examination. Because one of our purposes was to characterize normal SCGs, invasive procedures for confirmation were not acceptable; however, our results were consistent with those of anatomic reports. ${ }^{1-7}$ Also, as many participants as possible were examined to reveal the variations of morphology and location, minimizing the effects of misdetection for the ganglia and lymph nodes. Second, 2 different MR imaging instruments were used for this study because we assigned the specific protocols on separate instruments. The SCG signal was evaluated by thick sections of routine neck MR imaging. Evaluations using thin section thickness, such as neurography, might have been better to avoid partial volume effects and reveal detailed intraganglionic structures. Third, the lymph nodes that we classified into normal lymph nodes might have contained metastases, though there were significant differences in ADC values between normal and metastatic lymph node groups. Finally, we selected only adult cases. Our data might not be relevant to the pediatric population. In fact, the relative size of the SCGs and RPLNs may be different between adults and children because reactive retropharyngeal lymph nodes are more commonly seen in pediatric populations.

\section{CONCLUSIONS}

We have shown that MR imaging enables detection of SCGs. Although SCGs, IGVNs, and RPLNs showed similar shapes on cursory inspection, they can be differentiated individually by evaluating the signal intensity, size, and positional relationship. SCGs and RPLNs were on the lateral edges of the longus capitis muscle and the anteromedial side of the ICA, but RPLNs were smaller in volume, with a higher and more anteromedial position and lower contrast enhancement and ADC values than SCGs. IGVNs were smaller than SCGs and RPLNs and were located between the ICA and internal jugular vein. In other words, the RPLN, SCG, ICA, IGVN, and the internal jugular vein existed along a line from anteromedial to posterolateral on an axial section. Precise detection of them will lead to correct cancer staging and promote safe procedures such as lymph node biopsy and SCG block.

\section{REFERENCES}

1. Berkovitz B. Cervical sympathetic trunk. In: Standring S, ed. Gray's Anatomy: The Anatomical Basis of Clinical Practice. 39th ed. Edinburgh: Elsevier Churchill Livingstone; 2005:559-60

2. Fazliogullari Z, Kilic C, Karabulut AK, et al. A morphometric analysis of the superior cervical ganglion and its surrounding structures. Surg Radiol Anat 2016;38:299-302 CrossRef Medline

3. Yin Z, Yin J, Cai J, et al. Neuroanatomy and clinical analysis of the cervical sympathetic trunk and longus colli. J Biomed Res 2015;29: 501-07 CrossRef Medline

4. Civelek E, Karasu A, Cansever T, et al. Surgical anatomy of the cervical sympathetic trunk during anterolateral approach to cervical spine. Eur Spine J 2008;17:991-95 CrossRef Medline

5. Saylam CY, Ozgiray E, Orhan M, et al. Neuroanatomy of cervical sympathetic trunk: a cadaveric study. Clin Anat 2009;22:324-30 CrossRef Medline

AJNR Am J Neuroradiol 39:170-76 Jan 2018 www.ajnr.org 
6. Kiray A, Arman C, Naderi S, et al. Surgical anatomy of the cervical sympathetic trunk. Clin Anat 2005;18:179-85 CrossRef Medline

7. Wisco JJ, Stark ME, Safir I, et al. A heat map of superior cervical ganglion location relative to the common carotid artery bifurcation. Anesth Analg 2012;114:462-65 CrossRef Medline

8. Loke SC, Karandikar A, Ravanelli M, et al. Superior cervical ganglion mimicking retropharyngeal adenopathy in head and neck cancer patients: MRI features with anatomic, histologic, and surgical correlation. Neuroradiology 2016;58:45-50 CrossRef Medline

9. Lee JY, Lee JH, Song JS, et al. Superior cervical sympathetic ganglion: normal imaging appearance on 3T-MRI. Korean J Radiol 2016;17:657-63 CrossRef Medline

10. Hogan QH, Erickson SJ. MR imaging of the stellate ganglion: normal appearance. AJR Am J Roentgenol 1992;158:655-59 CrossRef Medline

11. Slappendel R, Thijssen HO, Crul BJ, et al. The stellate ganglion in magnetic resonance imaging: a quantification of the anatomic variability. Anesthesiology 1995;83:424-26 CrossRef Medline

12. Vargas MI, Viallon M, Nguyen D, et al. New approaches in imaging of the brachial plexus. Eur J Radiol 2010;74:403-10 CrossRef Medline

13. Mallouhi A, Marik W, Prayer D, et al. 3T MR tomography of the brachial plexus: structural and microstructural evaluation. Eur J Radiol 2012;81:2231-45 CrossRef Medline

14. Chhabra A, Thawait GK, Soldatos T, et al. High-resolution 3T MR neurography of the brachial plexus and its branches, with emphasis on 3D imaging. AJNR Am J Neuroradiol 2013;34:486-97 CrossRef Medline

15. Shibuya K, Sugiyama A, Ito S, et al. Reconstruction magnetic resonance neurography in chronic inflammatory demyelinating polyneuropathy. Ann Neurol 2015;77:333-37 CrossRef Medline

16. Zümre O, Salbacak A, Cicekcibasi AE, et al. Investigation of the bifurcation level of the common carotid artery and variations of the branches of the external carotid artery in human fetuses. Ann Anat 2005;187:361-69 CrossRef Medline

17. Faul F, Erdfelder E, Lang AG, et al. $G^{\star}$ Power 3: a flexible statistical power analysis program for the social, behavioral, and biomedical sciences. Behav Res Methods 2007;39:175-91 CrossRef Medline

18. Yuen HW, Goh CH, Tan TY. Enlarged cervical sympathetic ganglion: an unusual parapharyngeal space tumour. Singapore Med J 2006;47:321-23 Medline
19. Anil G, Tan TY. Imaging characteristics of schwannoma of the cervical sympathetic chain: a review of 12 cases. AJNR Am J Neuroradiol 2010;31:1408-12 CrossRef Medline

20. Costa NS, Salisbury SR, Donnelly LF. Retropharyngeal lymph nodes in children: a common imaging finding and potential source of misinterpretation. AJR Am J Roentgenol 2011;196:W433-37 CrossRef Medline

21. Mitsuoka K, Kikutani T, Sato I. Morphological relationship between the superior cervical ganglion and cervical nerves in Japanese cadaver donors. Brain Behav 2016 29;7:e00619 CrossRef Medline

22. Kiernan JA. Vascular permeability in the peripheral autonomic and somatic nervous systems: controversial aspects and comparisons with the blood-brain barrier. Microsc Res Tech 1996;35:122-36 Medline

23. Wendl CM, Müller S, Meier J, et al. High resolution contrast-enhanced ultrasound and 3-Tesla dynamic contrast-enhanced magnetic resonance imaging for the preoperative characterization of cervical lymph nodes: first results. Clin Hemorheol Microcirc 2012; 52:153-66 CrossRef Medline

24. Treggiari MM, Romand JA, Martin JB, et al. Cervical sympathetic block to reverse delayed ischemic neurological deficits after aneurysmal subarachnoid hemorrhage. Stroke 2003;34:961-67 CrossRef Medline

25. Siegenthaler A, Haug M, Eichenberger U, et al. Block of the superior cervical ganglion: description of a novel ultrasound-guided technique in human cadavers. Pain Med 2013;14:646-49 CrossRef Medline

26. Jankovic D. Superior cervical ganglion block. In: Jankovic D, Peng P, eds. Regional Nerve Blocks in Anesthesia and Pain Therapy. 4th ed. Cham: Springer; 2015:201-09

27. Elias M. Cervical sympathetic and stellate ganglion blocks. Pain Physician 2000;3:294-304 Medline

28. Wirz S, Wartenberg HC, Nadstawek J, et al. Superior cervical ganglion: an anatomical variant-are variations of the cranial carotid artery a risk factor for accidental intravascular injection? [in German]. Anaesthesist 2008;57:689-92 CrossRef Medline

29. Liu SS, Ngeow JE, Yadeau JT. Ultrasound-guided regional anesthesia and analgesia: a qualitative systematic review. Reg Anesth Pain Med 2009;34:47-59 CrossRef Medline 